\title{
Desempenho ocupacional em atividades de vida diária de pessoas com desnutrição crônica internadas em enfermarias de clínica médica
}

\author{
Lauren Machado Pinto ${ }^{a}$, Rita Aparecida Bernardi Pereira ${ }^{b}$, Aline Ferrari Fabric \\ ${ }^{a}$ Terapeuta ocupacional, Residente Multiprofissional em Atenção Hospitalar do Hospital de Clínicas, Área de \\ Concentração: Saúde do Adulto e Idoso, Universidade Federal do Paraná - UFPR, Curitiba, PR, Brasil \\ ${ }^{\text {b} T e r a p e u t a ~ o c u p a c i o n a l, ~ M e s t r e ~ e m ~ E d u c a c ̧ a ̃ o, ~ U n i v e r s i d a d e ~ F e d e r a l ~ d e ~ S a ̃ o ~ C a r l o s ~-~ U F S C a r, ~ S a ̃ o ~ C a r l o s, ~}$ \\ SP, Brasil. Professora Assistente do Departamento de Terapia Ocupacional, Setor de Ciências da Saúde, \\ Universidade Federal do Paraná - UFPR, Curitiba, PR, Brasil

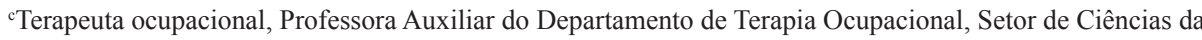 \\ Saúde, Universidade Federal do Paraná - UFPR, Curitiba, PR, Brasil
}

\begin{abstract}
Resumo: Introdução: A desnutrição configura-se como uma condição de saúde que provoca déficits no desempenho ocupacional. Objetivo: Realizar uma correlação entre o estado nutricional e desempenho ocupacional em atividades da vida diária (AVD), conforme grau do estado desnutricional. Metodologia: Estudo descritivo, exploratório, transversal, com avaliação de 50 pacientes com diagnóstico de desnutrição crônica internados em um hospital universitário na cidade de Curitiba, PR. Fez-se uso da medida de independência funcional (MIF) e de um questionário semiestruturado em que os sujeitos nomearam os três principais motivos definidos como limitadores do desempenho. O teste de Kruskall-Wallis foi utilizado para identificar a existência de diferença entre pelo menos dois dos grupos em análise, e o teste de comparações múltiplas, para verificar em quais pares de grupos existe diferença estatística significativa. Foi considerado o nível de significância igual a $95 \%(\mathrm{p} \leq 0,05)$. Resultados: No período de um ano de pesquisa foram avaliados 28 (56\%) pacientes do sexo feminino e 22 (44\%) do sexo masculino, com média de idade de 50,16 $( \pm 16,74)$ anos. Desses, $19(38 \%)$ em estado de desnutrição crônica leve; 16 (32\%) em estado de desnutrição crônica moderada; e 14 (28\%) em estado de desnutrição crônica grave. Foram identificadas alterações sobretudo em atividades avaliadas no uso da MIF motora, com mudanças relevantes no grau de dependência nos estados de desnutrição moderada a grave. As queixas dos pacientes foram ao encontro do que a literatura aponta como sintomas de desnutrição. Conclusão: O estudo comprova a relação entre a piora do estado nutricional e maior grau de dependência em AVDs.
\end{abstract}

Palavras-chave: Desnutrição, Terapia Ocupacional, Análise e Desempenho de Tarefas, Atividades Cotidianas.

\section{Occupational performance in daily life activities of subjects hospitalized because of chronic malnutrition}

\begin{abstract}
Introduction: Malnutrition is configured as a health condition that causes deficits in occupational performance. Objective: To establish a correlation between nutritional status and occupational performance in activities of daily life (ADLs) according to the desnutrition state level. Methodology: A descriptive, exploratory, cross-sectional evaluation of 50 patients diagnosed with chronic malnutrition admitted to a University Hospital in Curitiba, Parana state. We used the Functional Independence Measure (FIM) and a semi-structured questionnaire in which the subjects named the three main performance limiting reasons. The Kruskal-Wallis test was used
\end{abstract}

Autor para correspondência: Lauren Machado Pinto, Universidade Federal do Paraná, Rua Prof. José Moscalewski, 175, CEP 82100-310, Pilarzinho, PR, Brasil, e-mail: lauren.to@ hotmail.com

Recebido em 23/1/2012; Revisão em 9/11/2012; Aceito em 12/12/2012. 
to identify the existence of differences between at least two groups in the analysis, while the test of Multiple Comparisons was used to determine which pairs of groups showed statistically significant differences at $95 \%$ significance level $(p \leq 0.05)$. Results: We assessed 28 (56\%) female and $22(44 \%)$ male patients with mean age of $50.16( \pm 16.74)$ for one year. Out of these, $19(38 \%)$ presented mild chronic malnutrition, $16(32 \%)$ showed moderate chronic malnutrition, and $14(28 \%)$ presented severe chronic malnutrition. We identified changes particularly in activities evaluated in the use of motor FIM, with relevant alterations of dependence level for the states of moderate to severe malnutrition. Patients' complaints were in agreement with those pointed by the literature as symptoms of malnutrition. Conclusion: This study confirms the correlation between deterioration in nutritional status and high dependence level upon ADLs.

Keywords: Malnutrition, Occupational Therapy, Performance and Analysis Tasks, Daily Activities.

\section{Introdução}

A situação de doença e hospitalização caracteriza fatores que podem vir a restringir a capacidade funcional de sujeitos internados e, consequentemente, o seu desempenho ocupacional. Estudos têm demonstrado que outros fatores além desses podem vir a prejudicar esse desempenho, a caracterizar: idade avançada, múltiplas comorbidades, gravidade da condição clínica, estado nutricional, depressão, delirium, comprometimento cognitivo e físico, fatores ambientais e iatrogenias (CUNHA et al., 2009; REZENDE et al., 2004; SANTOS; ABREU, 2005). A desnutrição, enquanto condição de saúde merece destaque, levando-se em conta essa como uma síndrome multifatorial, a saber: primária, devido à ingestáo insuficiente de energia e proteínas; secundária, pela condição clínica do paciente; ou terciária quando adquirida ao longo da internaçáo (WAITZBERG, 2000). Ela predispóe a declínios da capacidade funcional, com aumento do tempo de internação, custos elevados ao serviço público, desestruturação da dinâmica familiar e incapacidade futura.

No entanto, as descrições empregadas para caracterizar os déficits dela advindos, sua gravidade, sua abrangência e quais atividades especificamente são prejudicadas não são discriminadas na literatura. A isso se aliam as diversas compreensões sobre o que seja capacidade funcional. A definiçáo de incapacidade é ampla e determinada por uma múltipla casuística, no qual fatores relacionados ao modelo biomédico, psicossocial e ambiental devem ser considerados (ALVES; LEITE; MACHADO, 2008).

Esse é o foco do terapeuta ocupacional que, como profissional da área da saúde, também estuda e favorece o desempenho ocupacional dos sujeitos em situação de internação. $\mathrm{Na}$ área hospitalar, o terapeuta ocupacional é o profissional responsável por minimizar a situação de ruptura do cotidiano e hospitalização, além de otimizar a capacidade funcional das pessoas, promovendo a maior independência possível (DE CARLO; BARTALOTTI; PALM, 2004).

A falta de detalhamento da relação entre desnutriçáo e desempenho ocupacional compromete uma compreensão mais abrangente do quadro clínico e, consequentemente, prejudica uma intervenção mais assertiva, que garanta melhores condiçóes de viabilizar a independência e autonomia dos sujeitos/ indivíduos. Além disso, subutiliza todo o potencial da equipe e dos familiares/cuidadores no tratamento.

Neste trabalho considerou-se a relação entre estado nutricional dos indivíduos sujeitos internados em unidade de clínica médica em um hospital universitário na cidade de Curitiba, PR, e déficits no seu desempenho ocupacional em atividades da vida diária. Levaram-se em conta também fatores relacionados ao sexo, idade, escolaridade, estado civil, ocupação atual, com fins descritivos do perfil epidemiológico da populaçáo. O objetivo deste estudo é analisar a variação da capacidade funcional nas atividades de vida diária (AVD) conforme o grau de desnutrição crônica do sujeito. Para complementar a análise, foram levados em conta os fatores considerados pelos sujeitos da pesquisa como principais limitadores ao desempenho independente. O projeto desenvolveu-se em parceria com o serviço de nutriçáo do hospital, responsável pelo diagnóstico de estado nutricional dos pacientes internados.

\section{Metodologia}

Trata-se de um estudo descritivo, transversal, prospectivo, desenvolvido a partir de projeto de pesquisa, com foco na análise do desempenho ocupacional de sujeitos internados em uma unidade de clínica médica com diagnóstico de desnutrição crônica. 
Anteriormente ao desenvolvimento prático do projeto realizou-se uma revisão de literatura a respeito da temática desnutrição, com uso de literatura clássica da clínica dessa condição de saúde, para maior compreensão da fisiopatologia e epidemiologia desse estado de desequilíbrio nutricional. A pesquisa incluiu busca em bases de dados da Biblioteca Regional de Medicina (BIREME), sendo que foram identificados estudos relacionados principalmente à Nutrição e Enfermagem em enfoque hospitalar, com apontamentos da epidemiologia da desnutrição, propostas de intervenção e atenção à saúde dessa população em diferentes contextos de hospitais universitários. Não foram encontrados estudos relacionados a intervençóes ou desenvolvimento de pesquisas por parte de terapeutas ocupacionais.

Conforme procedimento operacional padrão do hospital de referência deste estudo, foram considerados os estados de desnutrição aguda e crônica conforme o período de exposiçáo do sujeito a essa restrição: até 3 meses em fase aguda e mais que 3 meses de forma crônica. A percentagem de perda de massa corpórea também com potencial para defini-la de forma leve, moderada ou grave conforme perda inferior a $5 \%$; de $5 \%$ a $10 \%$; e superior a $10 \%$, respectivamente (WAITZBERG, 2000).

Foram selecionados para o estudo pacientes com diagnóstico de desnutrição crônica, homens e mulheres com idade superior a 18 anos, em níveis variados de escolaridade e estado civil, mediante assinatura de Termo de Consentimento Livre e Esclarecido (TCLE). Foram excluídos da pesquisa indivíduos com diagnóstico de doenças que afetam o sistema nervoso central e periférico. $\mathrm{O}$ total de sujeitos foi de 50 pacientes no período de 1 ano de pesquisa.

Os sujeitos selecionados foram avaliados no ambiente de enfermaria, sendo a medida de independência funcional (MIF) a avaliação escolhida para analisar o grau de assistência de que necessitavam nas atividades de vida diária (AVD). A MIF é um instrumento de avaliação da independência de sujeitos com incapacidades de origens variadas desenvolvido na década de 1980 nos Estados Unidos e validada no Brasil em 2000. Tem como objetivo principal avaliar de maneira quantitativa a carga de cuidados demandados por uma pessoa para as atividades motoras e cognitivas. A MIF é um instrumento originalmente desenvolvido para a avaliação em contexto hospitalar, através da entrevista e observaçáo direta do desempenho em atividades de vida diária analisadas nessa avaliação (RIBERTO et al., 2004). As atividades avaliadas pela MIF são: autocuidado, transferências, locomoção, controle esfincteriano, comunicação e cognição social, que inclui memória, interação social e resolução de problemas (RIBERTO et al., 2004). Para cada um desses itens é quantificado um escore de 1 a 7 , com a independência identificada de modo progressivo, conforme as etapas das atividades que o sujeito realiza com independência. A saber: 1 para dependência total (em que a pessoa realiza $0 \%$ ) da atividade; 2 para dependência máxima (sujeito realiza menos que $25 \%$ ); 3 para dependência moderada (pessoa realiza $50 \%$ da atividade); 4 para dependência mínima (pessoa realiza $75 \%$ da atividade); 5 para a necessidade de supervisão; 6 para a independência modificada (quando o sujeito faz uso de ajuda técnica ou necessita de um tempo maior para realizar a atividade); e 7 para a independência completa (quando a atividade é realizada em segurança, em tempo esperado).

Após a aplicação da MIF, foi solicitado que o sujeito citasse quais os três aspectos que considerava como maiores responsáveis para esses déficits no desempenho ocupacional, em ordem de importância. Essa pergunta foi realizada com base no objetivo específico desta pesquisa, para que fosse possível identificar fatores relacionados à clínica da desnutrição que justificassem o déficit no desempenho de atividades de vida diária.

\section{Considerações éticas}

O presente estudo foi aprovado pelo Comitê de Ética em Pesquisa em Seres Humanos da instituição de referência sob registro CAAE-0347.0.208.000-11. $\mathrm{Na}$ sua realizaçáa, todos os procedimentos éticos foram cumpridos.

\section{Resultados}

No período da pesquisa foram avaliados 50 sujeitos - 28 (56\%) do sexo feminino e 22 (44\%) do sexo masculino - com média de idade de 50,16 $( \pm 16,74)$ anos, média de escolaridade de 7,14 $( \pm 4,07)$ anos. Em relação ao estado matrimonial 9 (18\%) nunca tinham sido casados, 22 (44\%) eram na época casados, 2 (4\%) eram separados, 4 (8\%), divorciados, 9 (18\%), viúvos e $3(6 \%)$ viviam em coabitação. Quanto à ocupação no período: 7 (14\%) encontravam-se no desenvolvimento de emprego assalariado, $5(10 \%)$ trabalhavam por conta própria, 1 (2\%) trabalhava como voluntário (não assalariado), 14 (28\%) desenvolviam funçôes domésticas, 8 (16\%) eram aposentados, 3 (6\%) estavam desempregados e $1(2 \%)$ desenvolviam outro tipo de ocupação. $\mathrm{Na}$ classificação do estado nutricional, 19 (38\%) 
encontravam-se em estado de desnutrição crônica leve; 16 (32\%), em desnutrição crônica moderada e 14 (28\%), em desnutrição crônica grave.

A análise dos dados foi realizada utilizando o software R version 2.11.1 (AMARAL et al., 2010). Os dados quantitativos foram registrados através de medidas descritivas como médias (medida de tendência central) e desvio-padrão (medida de dispersão). O Quadro 1 apresenta as médias e desvio padrão de escores gerais da MIF, considerando todos os grupos da pesquisa de modo unitário. $\mathrm{O}$ teste de Kruskall-Wallis foi utilizado para identificar a existência de diferença entre pelo menos dois dos grupos em análise, e o teste de comparaçóes múltiplas, como complementar, foi utilizado para verificar em quais pares de grupos existia diferença estatística significativa. A realização desses testes objetivou identificar mudanças em escores da MIF conforme o estado nutricional dos sujeitos e realizar comparaçôes de mudanças significativas nesses escores conforme mudança naqueles estados. Para todos os testes realizados foi considerado nível de significância igual a 95\% ( $\mathrm{p} \leq 0,05)$. Assim, na análise descritiva dos dados, as seguintes médias gerais encontradas na MIF foram:

Nas probabilidades de alteraçôes nos escores conforme estado nutricional encontrou-se mudanças significativas relativas às atividades contempladas

Quadro 1. Média de escores gerais da MIF encontrada em sujeitos internados em unidade de clínica médica com diagnóstico de desnutrição.

\begin{tabular}{lcc}
\hline \multicolumn{1}{c}{ MIF } & Média & $\begin{array}{c}\text { Desvio } \\
\text { padrão }\end{array}$ \\
\hline Alimentação & 5,02 & 2,49481 \\
Higiene pessoal & 4,94 & 1,86712 \\
Banho & 4,16 & 1,76543 \\
Vestir metade superior & 4,96 & 2,02998 \\
Vestir metade inferior & 4,14 & 1,82958 \\
Utilizar vaso sanitário & 3,84 & 2,13197 \\
Controle da urina & 5,62 & 2,38096 \\
Controle das fezes & 5,76 & 2,42066 \\
Leito, cadeira e cadeira de rodas & 4,64 & 2,06783 \\
Vaso sanitário (mobilidade) & 4,04 & 2,20352 \\
Banheira e chuveiro & 4,18 & 2,01717 \\
Marcha & 4,32 & 2,07453 \\
Escadas & 2,86 & 2,25886 \\
Compreensão & 6,54 & 1,32803 \\
Expressão & 6,74 & 0,723089 \\
Interação social & 6,84 & 0,680936 \\
Resolução de problemas & 6,82 & 0,800255 \\
Memória & 6,82 & 0,800255 \\
\hline
\end{tabular}

na MIF motora, ou seja, autocuidado, controle esfincteriano e mobilidade. No entanto, atividades relacionadas à MIF cognitiva, compreensão, expressão, interação social, resolução de problemas e memória, não apresentaram mudanças significativas. No teste de comparações múltiplas entre os itens da MIF, ao nível de significância de $95 \%$, foi possível identificar que existe diferença estatisticamente significativa entre pelo menos um par de grupos, conforme identificado no Quadro 2. Para todos os itens identificados como MIF motora foi encontrado alteraçáo significativa, sobretudo nos estados de desnutrição moderada à crônica. Por outro lado, nos estados de desnutrição leve a moderada não houve alteraçôes. Os itens para avaliação cognitiva não apresentaram mudanças, independente do estado nutricional do sujeito.

Os sujeitos foram questionados quanto as fatores restritores do desempenho nas atividades avaliadas e apontaram os três que consideraram como limitadores em ordem hierárquica. Os motivos principais variaram, mas foram, sobretudo, dispnéia, dor, fadiga, fraqueza muscular e síncope. Outros motivos menos frequentes foram: envelhecimento, estados confusionais, falta de motivaçáo e medo de cair. O levantamento desses dados foi significativo para a identificação dos motivos pelos quais as alteraçôes no desempenho ocupacional nas atividades de vida diária fizeram-se presentes.

Os resultados encontrados indicaram um relevante déficit no desempenho ocupacional em atividades de vida diária (AVD) à medida que o estado nutricional dos indivíduos mostrava-se alterado, sobretudo na desnutrição crônica moderada e grave. Os escores médios da MIF indicaram alteraçôes principalmente em locomoção em escadas, mobilidade, uso do vaso sanitário e vestuário inferior, com necessidade maior de assistência para sua execução. $O$ controle esfincteriano e a alimentação mostraram-se comprometidos, com necessidade de supervisão dos cuidadores. Não se identificaram alteraçōes em funçôes cognitivas relacionadas a compreensão, expressão, interação social, solução de problemas e memória.

Resultado semelhante ao que aponta Matos (2004), que aplicou testes de avaliação cognitiva em 166 pacientes desnutridos crônicos internados na cidade do Porto, Portugal, sem evidências de disfunçôes cognitivas nessa população. Há relatos na literatura de que a desnutrição em crianças e idosos, principalmente, representa um fator de periculosidade para a integridade de funções cognitivas frente à fragilidade do sistema nervoso nessas duas populaçóes, com necessidades de cuidado 
Quadro 2. Alterações nos escores da MIF conforme estado nutricional de sujeitos internados em unidade de clínica médica com diagnóstico de desnutrição.

\begin{tabular}{lllllll}
\hline \multicolumn{1}{c}{ MIF } & Leve & $\begin{array}{l}\text { Desvio } \\
\text { padrão }\end{array}$ & Moderada & $\begin{array}{l}\text { Desvio } \\
\text { padrão }\end{array}$ & Grave & $\begin{array}{l}\text { Desvio } \\
\text { padrão }\end{array}$ \\
\hline Alimentação & 6,25 & 1,61815 & 5,625 & 2,36291 & 2,57143 & 1,98898 \\
Higiene pessoal & 6,25 & 1,01955 & 5,3125 & 1,25 & 2,64286 & 1,15073 \\
Banho & 5,2 & 1,23969 & 4,625 & 1,14746 & 2,14286 & 1,29241 \\
Vestir metade superior & 6,25 & 1,16416 & 5,625 & 1,31022 & 2,35714 & 1,08182 \\
Vestir metade inferior & 5,2 & 1,36111 & 4,6875 & 1,30224 & 2,0 & 0,960769 \\
Vaso sanitário & 5,3 & 1,21828 & 4,3125 & 1,77834 & 1,21429 & 0,578934 \\
Controle da urina & 6,9 & 0,447214 & 5,6875 & 2,21265 & 3,71429 & 2,99817 \\
Controle das fezes & 7,0 & 0,0 & 6,25 & 2,04939 & 3,42857 & 2,95386 \\
Mobilidade: leito, cadeira, cadeira de rodas & 5,85 & 1,1821 & 5,1875 & 1,60078 & 2,28571 & 1,5898 \\
Mobilidade: vaso sanitário & 5,5 & 1,4327 & 4,6875 & 1,49304 & 1,21429 & 0,578934 \\
Mobilidade: banheiro, chuveiro & 5,5 & 1,4327 & 4,5625 & 1,54785 & 1,85714 & 0,949262 \\
Marcha & 5,9 & 0,967906 & 4,5625 & 1,54785 & 1,78571 & 1,12171 \\
Escadas & 4,5 & 1,96013 & 2,4375 & 2,18994 & 1,0 & 0,0 \\
Compreensão & 6,7 & 1,34164 & 6,4375 & 1,63172 & 6,42857 & 0,937614 \\
Expressão & 6,9 & 0,447214 & 6,8125 & 0,75 & 6,42857 & 0,937614 \\
Interação social & 7,0 & 0,0 & 6,75 & 1,0 & 6,71429 & 0,726273 \\
Resolução de problemas & 7,0 & 0,0 & 6,6875 & 1,25 & 6,71429 & 0,726273 \\
Memória & 7,0 & 0,0 & 6,6875 & 1,25 & 6,71429 & 0,726273 \\
\hline
\end{tabular}

e intervenção (PORTO et al., 2010). Dessa forma, como as mudanças na capacidade funcional ficaram mais evidentes nas alteraçóes do estado nutricional (desnutrição crônica moderada ou grave), observa-se que o hospital pode vir a apresentar um papel relevante na promoção e prevenção em saúde com vistas à capacidade funcional desses sujeitos. Contudo, sem relatos desse tipo de experiência na literatura, conforme bases de dados da Bireme, evidencia-se a necessidade de desenvolvimento de estudos nesse quesito.

Assim, os resultados da pesquisa relacionados a atividades cotidianas justificam o encaminhamento, presença e intervençáo do terapeuta ocupacional no atendimento ao sujeito com desnutrição crônica. A importância de promover a independência vai além de açôes mecânicas de execução de tarefas, mas estabelece um processo para enriquecimento do cotidiano, com açóes significativas do sujeito sobre seu próprio corpo e cuidado (PEDRAL; BASTOS, 2008).

Nesta pesquisa, a MIF possibilitou identificar as demandas das atividades e os três principais motivos referenciados como responsáveis por comprometerem a capacidade funcional em cada atividade. Nesse último quesito é importante considerar que esses três aspectos vão ao encontro da fisiopatologia da situação de desnutrição, em que fadiga a esforços, dor e dispnéia são sintomas dessa condição de saúde (WAITZBERG, 2000). Como os terapeutas ocupacionais também podem intervir sobre as habilidades de desempenho necessárias às atividades, habilitando/capacitando/adaptando as possibilidades de participação, encontra-se nova justificativa para a intervenção desse profissional.

Na metodologia dessa intervenção, Lima, Gamallo e Oliveira (2010), em revisão de literatura, apontam mecanismos fisiopatológicos e terapêuticos na situação de desnutrição na infância, com sugestôes para tratamento da condição da desnutrição energética-protéica grave em crianças internadas. Neste estudo, o terapeuta ocupacional é citado enquanto profissional presente no tratamento desses pacientes, na estimulaçáo neuropsicomotora, com início já no tratamento em internação, com reforço ambulatorial. Todavia, ante o pequeno número de publicaçôes em que a atuação do profissional de Terapia Ocupacional com esta população é descrita - e tampouco são muitos os estudos com as populaçóes adulta e idosa -, faz-se necessário o desenvolvimento de outros estudos focados nessa prática, para sistematização e estabelecimento de metas de intervenção.

\section{Conclusão}

A realização de estudos que enfoquem a avaliação e intervenção com sujeitos em alterações do estado nutricional demonstra relevância significativa no 
contexto hospitalar. Estima-se que cerca de 30\% da população internada encontra-se em estado de desnutrição com maior mortalidade, tempo maior de internação, custos hospitalares (MATOS, 2004).

Este estudo mostrou a relaçáo entre déficit de desempenho ocupacional e desnutrição, sendo significativas as alterações nas fases moderada e grave. Apesar do número ainda reduzido de pesquisas realizadas nessa área por terapeutas ocupacionais, conclui-se finalmente a necessidade de desenvolvimento de outros estudos com essa temática, sobretudo com propostas de intervenção precoce e tardia, levando em consideraçáo que o terapeuta ocupacional é o profissional que tem entendimento dos pormenores das atividades, estando habilitado para intervir na melhora no desempenho ocupacional desses pacientes. Os resultados da pesquisa relacionados às atividades cotidianas justificam, pois, o encaminhamento, presença e intervenção do terapeuta ocupacional no atendimento ao sujeito com desnutrição crônica.

\section{Referências}

ALVES, L. C.; LEITE, I. C.; MACHADO, C. J. Conceituando e mensurando a incapacidade funcional na população idosa: uma revisão de literatura. Ciência e Saúde Coletiva, Rio de Janeiro, v. 13, n. 4, p. 1199-1207, 2008. http://dx.doi.org/10.1590/S1413-81232008000400016 AMARAL, M. R. S. et al. Software estatístico Livre R. Rio de Janeiro: UFRJ, 2010. Disponível em: <http:// www.ime.uerj.br/ -mrubens/slae/Texto_SLAE2.pdf>. Acesso em: 20 nov. 2012.

CUNHA, F. C. M. et al. Fatores que predispóem ao declínio funcional em idosos hospitalizados. Revista
Brasileira de Geriatria e Gerontologia, Rio de Janeiro, v. 12, n. 3, p. 475-487, 2009.

DE CARLO, M. R. P.; BARTALOTTI, C. C.; PALM, R. C. M. A Terapia Ocupacional em Reabilitação Física e Contextos Hospitalares: Fundamentos para a Prática. In: DE CARLO, M. M. P.; LUZO, M. C. M. Terapia Ocupacional: Reabilitaçáo Física e Contextos Hospitalares. São Paulo: Roca, 2004. p. 3-29.

LIMA, A. M.; GAMALLO, S. M. M.; OLIVEIRA, F. L. C. Desnutrição energetico-proteica grave durante a hospitalização: aspectos fisiopatológicos e terapêuticos. Revista Paulista de Pediatria, São Paulo, v. 28 , n. 3, p. 353-361, 2010. http://dx.doi.org/10.1590/ S0103-05822010000300015

MATOS, L. R. C. Desnutrição hospitalar no momento da admissão. 2004. 200 f. Dissertação (Mestrado em Ciências da Saúde)-Universidade do Porto, Cidade do Porto, 2004.

PEDRAL, C.; BASTOS, P. Terapia Ocupacional: Metodologia e prática. Rio de Janeiro: Rubio, 2008.

PORTO, J. A. et al. Efeitos da epilepsia e da desnutrição no sistema nervoso central em desenvolvimento: aspectos clínicos e evidências experimentais. Journal of Epilepsy and Clinical Neurophisiology, Porto Alegre, v. 16, n. 1, p. 26-31, 2010. http://dx.doi.org/10.1590/ S1676-26492010000100006

REZENDE, I. F. B. et al. Prevalência da desnutrição hospitalar em pacientes internados em um hospital filantrópico em Salvador (BA), Brasil. Revista de Ciências Médicas e Biológicas, Salvador, v. 3, n. 2, p. 194-200, 2004.

RIBERTO, M. et al. Validação da versão brasileira da Medida de Independência Funcional (MIF). Acta Fisiátrica, São Paulo, v. 11, n. 2, p. 72-76, 2004.

SANTOS, V. A.; ABREU, S. M. Impactos da desnutrição no paciente adulto hospitalizado. Revista de Enfermagem da UNISA, Santo Amaro, v. 6, p. 90-103, 2005.

WAITZBERG, D. L. Nutrição oral, enteral e parenteral na prática clínica. 3. ed. São Paulo: Atheneu, 2000.

\section{Contribuição dos Autores}

Lauren Machado Pinto: concepção e redação do texto manuscrito. Rita Aparecida Bernardi Pereira e Aline Ferrari Fabri: revisão e orientação. 